Proceedings of the 47th International School and Conference on the Physics of Semiconductors "Jaszowiec 2018"

\title{
Electron Spin Dephasing in a Double Quantum Dot Canceled via a Magnetic-Field Gradient
}

\author{
M. GAWEŁCZYK ${ }^{a, b, *}$ \\ ${ }^{a}$ Department of Theoretical Physics, Faculty of Fundamental Problems of Technology, \\ Wrocław University of Science and Technology, 50-370 Wrocław, Poland \\ ${ }^{b}$ Department of Experimental Physics, Faculty of Fundamental Problems of Technology, \\ Wrocław University of Science and Technology, 50-370 Wrocław, Poland
}

\begin{abstract}
Phonon-assisted tunneling of electrons between nonidentical quantum dots in a magnetic field has been recently shown to be affected by a spin pure dephasing. The process is due to the mismatch of the Zeeman splittings caused by the difference of effective electron $g$-factors in the two dots. Based on both analytical estimation and an accurate numerical modeling of a double quantum dot system, we show that this dephasing can be canceled by applying an appropriately tuned magnetic-field gradient that compensates for the built-in inequality of the Zeeman splittings.
\end{abstract}

DOI: 10.12693/APhysPolA.134.926

PACS/topics: 85.75.-d, 03.65.Yz

\section{Introduction}

It has been recently found that electron relaxation between orbital states characterized by unequal Zeeman splittings is affected by a pure dephasing of spin superposition $[1,2]$. The effect occurs without any direct spinenvironment coupling and is caused by a mismatch of energies dissipated during transitions involving the electron in the two spin states. It may be therefore interpreted as a which-way decoherence, with the observation leading to leakage of quantum information from the spin system affected by the phonon bath. The latter obtains some information on the spin state via the distinguishability of emitted phonons. This dephasing is expected to have pronounced consequences in the case of phonon-assisted tunneling between quantum dots (QDs) [2]. Transitions of this type can be used to dissociate optically generated electron-hole pairs, which suppresses their recombination [3]. In such a double QD (DQD) system the inequality of the Zeeman splittings in states localized in the two nonidentical dots stems from the mismatch of effective $g$-factors. The effect leads to a two-stage spin dephasing. The first one, present even at $T=0 \mathrm{~K}$, is a single loss of some amount of spin coherence exactly during tunneling. The remaining coherence $\mathcal{C}_{\infty}$ is given by [2]:

$$
\mathcal{C}_{\infty}=\mathcal{C}_{0} /\left(1-\mathrm{i} \Delta_{Z} / \hbar \bar{\Gamma}\right)
$$

where $\mathcal{C}_{0}$ is the initial spin coherence, $\Delta_{Z}$ is the difference of the Zeeman splittings in the two QDs, and $\bar{\Gamma}=\left(\Gamma_{-}+\Gamma_{+}\right) / 2$ is the tunneling rate averaged over spin states. Additionally, at $T>0 \mathrm{~K}$, due to repetitive virtual tunneling, the effect accumulates and leads to an exponential loss of coherence preserved after tunneling with dephasing time $\tau_{d}$ given by [2]:

\footnotetext{
*e-mail: michal.gawelczyk@pwr.edu.pl
}

$\frac{2}{\tau_{d}}=\bar{\Gamma}+\Gamma_{v} \operatorname{Re} \sqrt{\left(\bar{\Gamma}+\Gamma_{v}\right)^{2}+2 \mathrm{i} \omega_{Z}\left(\Gamma_{v}-\bar{\Gamma}\right)-\omega_{Z}^{2}}$,

where $\omega_{Z}=\Delta_{Z} / \hbar, \Gamma_{v} \simeq \bar{\Gamma} \mathrm{e}^{-\Delta / k T}$, and $\Delta$ is the electron energy difference between QDs at $B=0 \mathrm{~T}$. Due to this, not only electrons that actually tunnel are subject to spin dephasing, but also those statically localized in tunnel-coupled structures, which are utilized currently in quantum information processing [4]. Hence, limiting the discussed process is of particular interest. While it is possible to minimize $\Delta_{Z}$ by properly designing the structure [2], unavoidable technological inaccuracies are enough to significantly limit spin coherence.

Here, we consider a DQD system in an in-plane magnetic field $\boldsymbol{B}=B \widehat{x}$ with the electron prepared in the $z$-axis spin-up state $|\uparrow\rangle$ and propose to use an additional slanted field to cancel spin dephasing. Using numerical model of a realistic InGaAs system, we determine the gradient needed for this to be experimentally achievable.

\section{System and analytical estimations}

We begin with the fact that a magnetic-field gradient

$$
b=\partial B_{x}(\boldsymbol{r}) / \partial z,
$$

resulting in a difference of field magnitudes at positions of the two QDs, contributes to the mismatch of the Zeeman splittings in an analogous manner as the difference of $g$-factors does. Thus, it should be possible to compensate for the built-in $\Delta_{Z}$ by applying the magnetic field with appropriately tuned gradient along the $z$-axis. For a DQD system characterized by Landé factors $g_{1}$ and $g_{2}$ and the interdot distance $D$, subject to the magnetic field with the homogeneous component of magnitude $B_{0}$, the optimal gradient may be found to be

$$
b_{\mathrm{opt}} \simeq 2 \frac{B_{0}}{D} \frac{g_{1}-g_{2}}{g_{1}+g_{2}} .
$$


This would be exact for point-like QDs and possible inaccuracy stems from a finite extent of wave functions along the $z$-axis. The latter is however typically much less than $D$, hence Eq. (4) may prove to be a good approximation.

In Fig. 1, we present a general energy diagram of the system. On the left, one may find two spin-degenerate orbital levels $\epsilon_{i}^{(o)}$ corresponding to the electron wave function localized in each of the QDs, separated by the energy difference $\Delta$. In the middle, the in-plane magnetic field lifts the degeneracy by unequal Zeeman splittings $\epsilon_{i}^{(Z)}=g_{i} \mu_{\{\mathrm{B}} B$, which results in different transition energies for tunneling involving the two spin states, $\hbar \omega_{ \pm}=\Delta \pm \Delta_{Z} / 2$, where $\Delta_{Z}=\epsilon_{2}^{(Z)}-\epsilon_{1}^{(Z)}$. Finally, the diagram on the right corresponds to the system subject to the additional slanting magnetic-field component that cancels $\Delta_{Z}$. Dashed arrows mark tunneling transitions, while wavy arrows correspond to emitted phonons with given frequencies.

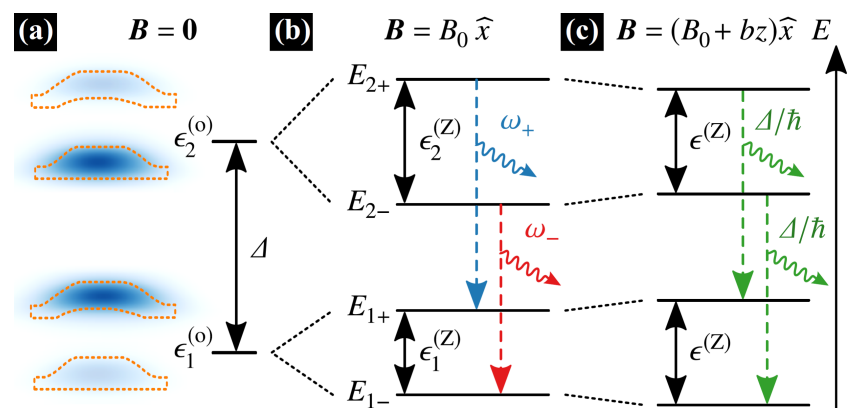

Fig. 1. Energy levels in the system: (a) no magnetic field, (b) in-plane field $B$, (c) in-plane field with added optimal gradient. Dashed arrows mark tunneling transitions with rates $\Gamma_{ \pm}$, wavy arrows depict emitted phonons with frequencies $\omega_{ \pm}$.

\section{Numerical modeling}

To verify our idea of canceling $\Delta_{Z}$ and the resulting spin dephasing as well as to estimate the magnetic-field gradient needed for this in the case of a typical system, we perform an accurate numerical modeling of a DQD structure. The modeled system consists of two vertically stacked, slightly misaligned, $\operatorname{In}_{x} \mathrm{Ga}_{1-x}$ As dome-shaped QDs with an intradot gradient of In content $[5,6]$ and deformation of the base of the upper dot [7]. We add randomness to the material distribution and perform Gaussian averaging with the standard deviation of $9 \AA$ to simulate the material intermixing. A cross-section of the resulting material composition is presented in Fig. 2 along with basic parameters of the structure. The dots differ in geometry and material composition, which represents typical deviations met in their self-assembly [7].

Electron eigenstates are calculated in the 8-band envelope-function $\boldsymbol{k} \cdot \boldsymbol{p}$ theory [8-10]. Numerical implementation used [11] includes spin-orbit effects [12, 13], external electric and magnetic fields [14], as well as strain $[15,16]$ and the resulting nonlinear piezoelectric

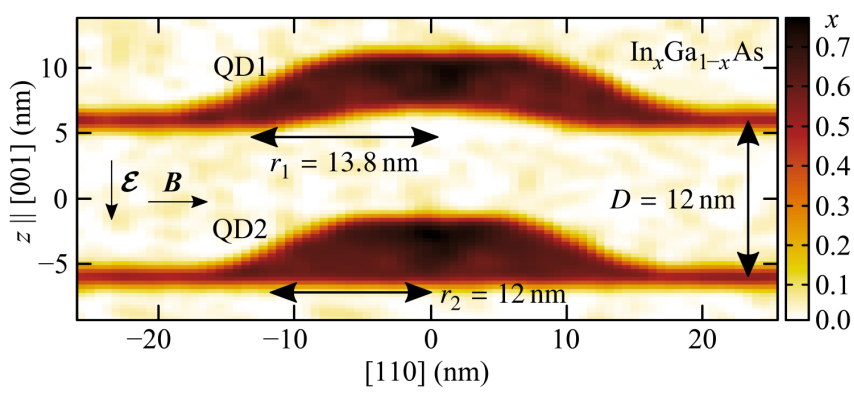

Fig. 2. A cross-section of the material composition (color gradient) of the double QD structure in the (1 $\overline{10})$ plane.

field [17]. The axial electric field $\mathcal{E}$ is used to tune the electron delocalization between QDs. Four lowest-energy states denoted $|1-\rangle,|1+\rangle,|2-\rangle$, and $|2+\rangle$ with energies $E_{1(2) \pm}$ are computed and form the basis $\mathcal{B}$ for further calculations. Here, $| \pm\rangle=(|\downarrow\rangle \pm|\uparrow\rangle) / \sqrt{2}$ are the Zeeman eigenstates in the in-plane magnetic field expressed in $z$-axis spin states, while the number indicates the QD where the electron is mostly localized. The calculated electron density in the two orbital states is presented in projection onto the $(1 \overline{1} 0)$ plane in the left part of Fig. 1. Calculating electron energies in the magnetic field, we find the $g$-factors in the two dots. The average value $\bar{g} \approx 1.19$ is in a reasonable agreement with experimental data [18], and the mismatch is $\Delta g=g_{2}-g_{1}=0.0189$. According to Eq. (4), for $B=1 \mathrm{~T}$ this corresponds to $b_{\text {opt }} \approx-1.47 \mathrm{mT} / \mathrm{nm}$, which is comparable to the values (up to $\sim 2 \mathrm{mT} / \mathrm{nm}$ ) obtained recently with micromagnets [19].

To find the transition rates $\Gamma_{ \pm}$, we include the coupling to the acoustic-phonon bath,

$H_{\mathrm{int}}=\sum_{i j \in \mathcal{B}} \sigma_{i j} \int \mathrm{d}^{3} \boldsymbol{r} \boldsymbol{\Psi}_{i}^{\dagger}(\boldsymbol{r})\left[H_{B-P}^{(\mathrm{ph})}(\boldsymbol{r})+V_{p}(\boldsymbol{r})\right] \boldsymbol{\Psi}_{j} \boldsymbol{r}$,

where $\sigma_{i j}=|i\rangle\langle j|, \quad \boldsymbol{\Psi}_{i}$ is a pseudo-spinor of the $i$-th eigenstate envelope functions, $H_{B-P}^{(\mathrm{ph})}$ is the Bir-Pikus Hamiltonian [15] evaluated with the phonon-induced strain field [20, 21], and $V_{p}$ is the piezoelectric potential also induced by the latter. The tunneling rates for the two spin states are then found from the Fermi golden rule,

$$
\Gamma_{ \pm}=2 \pi R_{i j j i}\left(\omega_{j i}\right) ; \quad(i, j)=(2 \pm, 1 \pm),
$$

where $R_{i j k l}(\omega)=|n(-\omega)| \sum_{\boldsymbol{q} \lambda} H_{\mathrm{int}}^{(i j)} H_{\mathrm{int}}^{(k l)} \delta\left(|\omega|-\omega_{\boldsymbol{q} \lambda}\right) / \hbar^{2}$ are phonon spectral densities [22], $\hbar \omega_{i j}=E_{j}-E_{i}, n(\omega)$ is the Bose distribution, $H_{\mathrm{int}}^{(i j)}=\left\langle i\left|H_{\mathrm{int}}\right| j\right\rangle$, and $\omega_{\boldsymbol{q} \lambda}$ is the frequency of $\lambda$-branch phonons with wave vector $\boldsymbol{q}$. The calculated values are $\Gamma_{-}=0.499 \mathrm{~ns}^{-1}$ and $\Gamma_{+}=0.492 \mathrm{~ns}^{-1}$. This, via Eq. (1), gives the preserved coherence $\mathcal{C}_{\infty} / \mathcal{C}_{0}=0.286$ and 0.0595 at $B=1 \mathrm{~T}$ and $5 \mathrm{~T}$, respectively. Thus, we deal with a significant coherence loss, destructive from the point of view of applications in quantum information. Additionally, the finitetemperature dephasing times, Eq. (2), are $4.92 \mu$ s at $T=5 \mathrm{~K}$ and $13.2 \mathrm{~ns}$ at $T=20 \mathrm{~K}$. 
To check the effectiveness of the proposed decoherence canceling method, electron states in the slanting magnetic field are calculated. In Fig. 3, we present the results of a series of calculations with varying $b$ for $B_{0}=1 \mathrm{~T}$. In part (a) one may notice that, as expected, the Zeeman splittings (dashed lines) for the two orbital states cross, hence $\Delta_{Z}$ vanishes, close to the predicted gradient value $b_{\text {opt }}$, at $b_{\text {opt }}^{\text {(num })} \approx-1.32 \mathrm{mT} / \mathrm{nm}$. This corresponds to the maximum in the plot of preserved coherence (solid line) calculated analytically according to Eq. (1). At the maximum, $\mathcal{C}_{\infty} / \mathcal{C}_{0} \approx 1$, which means no coherence loss during tunneling. Additionally, part (b) shows that post-tunneling dephasing time diverges at $b_{\mathrm{opt}}^{\text {(num) }}$.

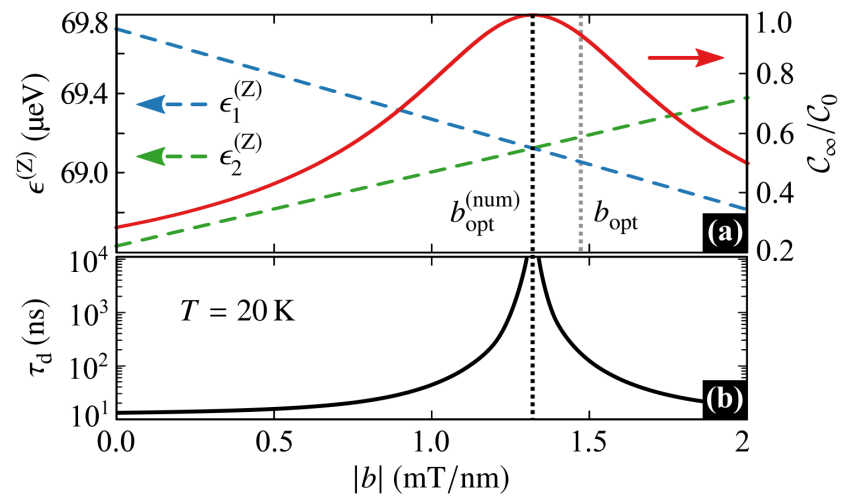

Fig. 3. The magnetic-field gradient $b$ dependence of (a) Zeeman splittings $\epsilon_{i}^{(Z)}$ (dashed lines, left axis) in the two QDs and the estimated preserved spin coherence $\mathcal{C}_{\infty}$ (solid line, right axis), (b) dephasing time $\tau_{d}$ at $T=$ 20 K. $B_{0}=1 \mathrm{~T}$.

To verify this, we model the phonon-induced dissipative electron evolution with a non-secular Markovian Redfield equation [23] for the reduced density matrix, written in the interaction picture, $\dot{\rho}(t)=\mathcal{L}[\rho(t)]$, where

$\mathcal{L}[\rho]=\pi \sum_{i j k l \in \mathcal{B}} \mathrm{e}^{\mathrm{i}\left(\omega_{i j}-\omega_{k l}\right) t} R_{j i k l}\left(\omega_{k l}\right)\left[\sigma_{k l} \rho \sigma_{i j}^{\dagger}-\sigma_{i j}^{\dagger} \sigma_{k l} \rho\right]+$ h.c.

Solving it numerically with the initial state $\rho(0)=$ $|2 \uparrow\rangle\langle 2 \uparrow|$, we obtain the evolution of spin coherence $\mathcal{C}(t)=\mid \sum_{i}\langle i-|\rho(t)| i+\rangle$. For $b=b_{\text {opt }}^{\text {(num) }}$, the value of spin coherence after 10 tunneling times is $\mathcal{C}(10 / \bar{\Gamma})=$ 0.4997 , which confirms the suppression of decoherence. Regardless of temperature, there is also no observable post-tunneling dephasing in the computationally available time scale.

\section{Conclusions}

We have shown that an appropriately tuned magneticfield gradient may be used to cancel pure spin dephasing that affects electrons during non-resonant tunneling in a double QD system in the magnetic field. The dephasing results from the inequality of energies dissipated in the case of transitions involving the two spin states. In a double quantum dot system, this is due to the unavoidable nonidentity of the dots leading to the difference between local effective $g$-factors. The resulting mismatch of the Zeeman splittings may be, however, compensated for with use of a magnetic-field gradient. We have analytically estimated the optimal slope of the field and then checked it for a realistically modeled structure showing that the difference of the Zeeman splittings may actually be canceled. Simulating the phonon-driven dissipative evolution of the system with a quantum master equation, we have found that in such a tuned system spin coherence is fully preserved during tunneling. The value of gradient needed in the case of simulated nonidentical quantum dots has been found to be technologically achievable for moderate magnitudes of the uniform magnetic field.

\section{Acknowledgments}

Support from the Polish National Science Centre by Grant No. 2014/13/B/ST3/04603 is acknowledged. Calculations have been carried out using resources provided by Wroclaw Centre for Networking and Supercomputing (http://wcss.pl), Grant No. 203. I am grateful to Krzysztof Gawarecki for sharing his implementation of the $\boldsymbol{k} \cdot \boldsymbol{p}$ method and carefully reading the manuscript as well as to Paweł Machnikowski for valuable discussions.

\section{References}

[1] M. Krzykowski, M. Gawełczyk, P. Machnikowski, Acta Phys. Pol. A 130, 1165 (2016).

[2] M. Gawełczyk, M. Krzykowski, K. Gawarecki, P. Machnikowski, Phys. Rev. B 98, 075403 (2018).

[3] M. Gawełczyk, P. Machnikowski, Semicond. Sci. Technol. 32, 045005 (2017).

[4] D. Kim, S.G. Carter, A. Greilich, A.S. Bracker, D. Gammon, Nature Phys. 7, 223 (2011).

[5] M.A. Migliorato, A.G. Cullis, M. Fearn, J.H. Jefferson, Phys. Rev. B 65, 115316 (2002).

[6] V. Jovanov, T. Eissfeller, S. Kapfinger, E.C. Clark, F. Klotz, M. Bichler, J.G. Keizer, P.M. Koenraad, M.S. Brandt, G. Abstreiter, J.J. Finley, Phys. Rev. B 85, 165433 (2012).

[7] H.J. Krenner, S. Stufler, M. Sabathil, E.C. Clark, P. Ester, M. Bichler, G. Abstreiter, J.J. Finley, A. Zrenner, New J. Phys. 7, 184 (2005).

[8] M.G. Burt, J. Phys. Condens. Matter 4, 6651 (1992).

[9] B.A. Foreman, Phys. Rev. B 48, 4964 (1993).

[10] T.B. Bahder, Phys. Rev. B 41, 11992 (1990).

[11] K. Gawarecki, P. Machnikowski, T. Kuhn, Phys. Rev. B 90, 085437 (2014).

[12] R. Winkler, Spin-Orbit Coupling Effects in TwoDimensional Electron and Hole Systems, Springer, Berlin 2003.

[13] K. Gawarecki, Phys. Rev. B 97, 235408 (2018).

[14] T. Andlauer, R. Morschl, P. Vogl, Phys. Rev. B 78, 075317 (2008).

[15] G. Bir, G. Pikus, Symmetry and Strain-Induced Effects in Semiconductors, Wiley, 1974. 
[16] C. Pryor, Phys. Rev. B 57, 7190 (1998).

[17] M.A. Caro, S. Schulz, E.P. O'Reilly, Phys. Rev. B 91, 075203 (2015).

[18] Y. Toda, S. Shinomori, K. Suzuki, Y. Arakawa, Appl. Phys. Lett. 73, 517 (1998).

[19] J. Yoneda, T. Otsuka, T. Takakura, M. PioroLadrière, R. Brunner, H. Lu, T. Nakajima, T. Obata, A. Noiri, C.J. Palmstrøm, A.C. Gossard, S. Tarucha, Appl. Phys. Expr. 8, 084401 (2015).
[20] L.M. Woods, T.L. Reinecke, R. Kotlyar, Phys. Rev. B 69, 125330 (2004).

[21] K. Roszak, V.M. Axt, T. Kuhn, P. Machnikowski, Phys. Rev. B 76, 195324 (2007).

[22] K. Roszak, A. Grodecka, P. Machnikowski, T. Kuhn, Phys. Rev. B 71, 195333 (2005).

[23] F. Breuer, H. Petruccione, The Theory of Open Quantum Systems, Oxford University Press, 2007. 